\title{
EN TORNO A ne- COMO PRIMER ELEMENTO DE UNA SERIE DE VOCABLOS CON SENTIDO NEGATIVO
}

Having studied in a previous paper the Latin composita with in- the authoress analyzes such composita with ne- as nego, negotium and nefas and draws some conclusions on the negative prefixes in general.

F. Bader, al estudiar los compuestos nominales del latín ${ }^{1}$, menciona un tipo particular de "yuxtapuestos" constituido por aquellos cuyo primer elemento es ne-, partícula que entra en la formación de pronombres (nemo) y nombres diversos. Algunos de estos nombres son deverbativos (nescius < ne scio), y otros proceden de una frase nominal (nefas, necesse, nimirum), ya que no parece haber verdaderos yuxtapuestos nominales ${ }^{2}$.

Estas consideraciones, halladas cuando nos encontrábamos estudiando los usos y valores del prefijo negativo in- ${ }^{3}$, llamaron nuestra atención por lo que podfan tener de interés complementario para aquel trabajo. Volvimos a encontrar algunas referencias al mismo tema al consultar, por el mismo motivo, un artículo de T. González a propósito de nego y negotium 4 .

Las conclusiones obtenidas del estudio de in-nos ponen ahora en situación de contemplar el problema de $n e$ - desde una nueva perspectiva, si bien no pretendemos en modo alguno resolver los problemas

1 F. Bader, La formation des composés nominaux du latin, Paris, 1962.

2 Cf. Bader, pp. 37 y 302 .

3 El prefijo in- ha sido el tema de nuestra tesis doctoral (*El prefijo in-: Un estudio sobre los antónimos en latín", Santiago de Compostela, 1975) que, reformada y ampliada, está en trance de publicación.

- T. González, "Aio y nego, otium, auus y negotium y la teoría laringalo, Cuadernos de Filología Cldsica I, 1971, pp. 21 1-222. Véase K. Brugmann, "Die lateinische Partikel ne ('nicht') in 'Zusammensetzung mit anlautenden Wörtern., I F 6,1896 , p. 80 y ss. Añádase a esto las referencias que hacen A. Meillet y J. Vendryes, Traité de grammaire comparée des langues classiques, Paris, 1948, p. 434 . 
planteados por la etimología de ciertas formaciones en las que interviene, sino simplemente arrojar una nueva luz sobre el tema al proprocionar una visión de conjunto que agrupe de algún modo los datos dispersos ${ }^{1}$.

I.

La negación indoeuropea ${ }^{*} n$ - aparece en latín bajo su forma plena como ne-, y en grado cero como in- 2 .

En forma libre se utiliza $n \bar{e}$, que en principio parece no haber tenido ningún valor subordinante; pero, en el reparto que la lengua ha hecho de las diversas partículas negativas, le ha correspondido la expresión de la prohibición, restricción, etc., acompañando generalmente al imperativo y al subjuntivo ${ }^{3}$. Por el contrario, la variante $n e \check{~ n o ~ s e ~ e n c u e n-~}$ tra aislada, sino como primer término de algunas palabras, que forman una serie limitada en cuanto que esta posibilidad de formación sólo existía en época antigua.

2.

Atendiendo a la base sobre la que han sido formados los vocablos en los que aparece $n e-$, pueden establecerse los siguientes grupos:

$$
\begin{array}{ll}
\text { I. } & n e+\text { verbos: } \mathrm{I} .>\text { verbos } \\
2 . & >\text { adjetivos } \\
\text { 2. } & n e+\text { partículas } \\
\text { 3. } & n e+\text { pronombres } \\
\text { 4. } & n e+\text { sustantivos }
\end{array}
$$

1 Por ello no profundizamos tampoco de un modo especial en la histcria concreta de cada palabra y nos limitamos a exponer tan sólo los datos que juzgamos de mayor interés.

- El prefijo negativo por excelencia, del que simplemente haremos mención en cuanto a las relaciones que mantiene con $n e$ -

3 No nos interesa aqui estudiar las funciones sintácticas de esta partícula, sino su papel como primer miembro de "yuxtapuestos" (utilizando la denominación de Bader). 


\section{I. $\mathrm{Ne}+$ verbos}

\section{I.I. $N e+$ verbos $>$ verbos}

Desde época muy temprana se encuentran en latín una serie de verbos (nego, nescio, neglego, etc.) en cuyo primer elemento es reconocible la partícula que nos ocupa.

Nego presenta dificultades en cuanto a su origen, que ha querido ponerse en relación con $a g o^{1}$ y con aio ${ }^{2}$. Efectivamente, nego se contrapone a aio en algunos contextos (como, por ejemplo, Plaut. Rud. 430, uel tu mihi a i as, uel ne ges); esta oposición es real en cuanto al significado, pero ello no quiere decir que tenga que serlo también en cuanto a la forma. De hecho, Walde y Hofmann ${ }^{3}$ - al igual que Eirnout y Meillet - prefieren partir de la consideración de que lo que ha dado lugar al verbo es la forma ${ }^{*} n e g(i)$ de la negación ${ }^{5}$.

Nescio es la negación, lexical y gramatical, de scio (cf., por ejemplo, Plaut., Merc. 382, hunc nescire sat scio); ne debe de haber sido en principio independiente de scio, pero, debido a la frecuencia con que probablemente se emplearía este verbo en forma negativa, terminó por fundirse con él.

Lo mismo habría ocurrido con nolo ${ }^{6}$ ("ne uolo $>{ }^{*}$ nowolo $>$ nolo), como lo prueba el hecho de que, en un primer momento, todavia llevaba ne en las demás personas (neuis, neuolt) ${ }^{7}$; las formas con non son más recientes y responden tal vez a la tendencia a regularizar los paradigmas, en este caso a partir de nolo, donde la $o$ se explica, como acabamos de ver, por la evolución fonética ante uolo. Además, estas formas nuevas

1 Vid., por ejemplo, A. Forcellini, Totius latinitatis lexicon, Patavii, 1940, III, p. 354 .

2 年. González (art. cit.), entre otros, establece como punto de partida para nego la misma raíz que para aio, explicando la evolución de ambos verbos merced a la teoria de las laringales.

3 Cf. A. Walde-J. B. Hofmann, Lateinisches Etymologisches Wörterbuch (=LEW), Heidelberg, 1938-56, II, s. u. nego.

4 Cf. A. Ernout-A. Meillet, Dictionnaire étymologique de la langue latine. Histoire des mots (=DELL), Paris, 1964 , s. u. nego.

- F. Bader acepta esta hipótesis como más probable, explicando la forma * neg como una variante combinatoria de nec, existente también en estado libre (cf. Bader, p. 39).

- Aparece a menudo opuesto a uolo, como en Plaut., Epid. 595: ubi u o les pater esse, ibi esto: ubi noles, ne fueris pater.

7 Vid., por ejemplo, Plaut., Trin. 1156: Filiam meam tibi desponsatam esse audio: Nisi tu neuis. 
no han logrado ya fundirse con el verbo, sino que se mantienen como unidades distintas: non uis, non uult, etc. ${ }^{1}$ (cf., en cambio, sis < $<e(i)$ $(u)$ is 'si quieres', sultis < se(i) (u)ultis 'si quereis', conservadas tan sólo como formas de cortesía).

La frecuencia de aparición de queo en secuencia negativa lleva, entre otros motivos, a Ernout y Meillet ${ }^{2}$ a presentar como aceptable la hipótesis de que para la formación de queo y nequeo hay que partir de una forma impersonal nequitur 'esto no va' procedente de neque + itur; es decir, de nequitur se habría extraído nequeo, y luego éste sería comprendido como 'non queo' (por analogía con nescio, etc.), por lo que la aparición de queo como retrogradación no resulta extraña.

Neglego, con sus variantes neclego y negligo ${ }^{3}$, deben de proceder de la fusión de la partícula negativa con el verbo lego, aun cuando su posterior lexicalización dificulta el establecimiento de una oposición semántica 4 .

\section{I.2. $\mathrm{Ne}+$ verbos $>$ adjetivos}

Paralelamente a formas como neglegens (o negligens) y neglectus, aparecen - a través de *nec opinor-necopinans y necopinatus y, a su lado, un necopinus que podría hacer pensar en un adjetivo *opinus en relación con opinor. Sin embargo, necopinus no está atestiguado hasta Ovidio (como su paralelo inopinus lo está sólo desde Virgilio), por lo que parece necesario pensar en una retrogradación a partir de necopinans, necopinatus ${ }^{5}$.

Nescius es un adjetivo derivado de nescio (como scius $<$ scio), que

1 Cf. DELL s. u. uolo. Ibidem: La forma del imperativo, noli, reciente y formada sobre el subjuntivo, se emplea casi como una partícula para indicar una prohibición cortés, atenuada: noli facere (/v! uelim facias).

- Cf. $D E L L$, s. u. queo; también alli puede verse, respecto al valor de este verbo, la oposición en matices con possum.

- La presencia de la apofonia en negligo, negligens, es un argumento más a favor de la antigïedad de la formación.

- Lego 'leer', ni siquiera como 'recoger, reunir, elegir', no parece tener un antónimo, más que de un modo bastante relativo, en neglego 'descuidar, menospreciar'.

- El LELL (s. u. opinor) da como una posibilidad que inopinus y necopinus proceden de opinio (cf. gr. $\delta \delta \xi \alpha / \& \delta \circ \xi \circ)$ ); pero, puesto que nec se encuentra exclusivamente en formaciones antiguas, parece más verosimil la teoría de la retrogradación (que es, por otra parte, la que admite Bader, p. 203). 
forma serie con conscius, praescius, inscius ${ }^{1}$. Por el mismo procedimiento -y también quizás por analogía con el anterior-- se ha formado nesap $(i) u^{2}$ a partir de sapio, aunque no exista ${ }^{*}$ nesapio.

Nefandus y nefans derivan de fari, por influjo de (ne)fas ${ }^{3}$.

Nefrens, por su parte, es un adjetivo un tanto extraño, que aparece en una glosa de Paulo Festo ${ }^{4}$ y que debe de derivar de frendo ('rechinar los dientes' $>$ 'triturar') ${ }^{5}$.

Necesse (necessum, necessus) se emplea con sum y habeo para formar las locuciones del tipo necesse est/habeo, que marcan una necesidad

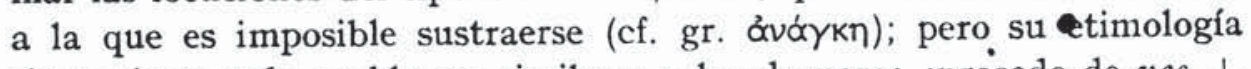
sigue planteando problemas similares a los de nego: ¿procede de nec + esse? ¿O de ne + cedo (tal vez de un sustantivo *cessis 'no hay modo de retroceder')? ${ }^{6}$.

1 Inscius parece significar lo mismo que nescius, si bien en el segundo quizás esté presente un mati\% peyorativo ('necio') que no existe en inscius. De todas formas, el proceso de formación es distinto, ya que, mientras nescius deriva de nescio, inscius está creado sobre scius.

2 Nesapius está en Petronio (cf. Forcellini III, p. 363), y nesapus en Terenciano Escauro (cf. D. du Cange, Glossarium mediae et infimae latinitatis, Paris, $1840-50$, I, p. 622). Forcellini propone para esta cita de T. E. la lectura nesapius, igual a la de Petronio.

- Nefans es empleado en plural neutro (nefantia) con el sentido de nefanda, por I,ucilio y Varrón: cf. DELL, s. u. for. Cf. también infans e infandus, en los que -aparte de la lexicalización del primero-- está más claro el valor de for, al no percibirse ninguna relación con nefas.

- I a glosa confunde dos palabras: un adjetivo que significa 'sin dientes, que no puede morder', y un sustantivo (gr. veфpoús) 'riñones'. Cf. $L E W$ II, s. u. nefrens; DELL, s. u. nefrendes; Forcellini III, p. 353. Véase también lo que dice al respecto A. Pariente, "Más sobre 'nebrundines't, Emerita 11, 1943, pp. 338411 .

- De ser correcta la lectura neparcus (explicada como non parcus) en Plaut., Psertd. 77 (cf. Forcellini III, p. 359), habria que pensar en la posibilidad de que ne se hubiese utilizado también para negar adjetivos; pero lo que allí dice es más bien neparcunt o reparcunt.

También en una glosa de Paulo lesto aparece nepus $==$ non purus, que Pariente cree que debe de ser reducido a nepos (cf. A Pariente, "En torno a 'nepos'", Emerita 11, 1943, Pp. 60-122).

- Cf. DELL, s. u. necesse; LEW II, s. u. necesse; Forcellini III, p. 349: A. Pariente, "Necesse», Emerita 43, 1975, pp. 25-40. 


\subsection{Ne + partículas}

\subsection{I.}

Al lado de $n e, n e c$, como partícula independiente, existen formas reforzadas de la negación (recuérdese que ne se especificó como partícula subordinante), que pueden proceder de $n \bar{e}$ o $n \check{e}$, como son neque (cf. atque - ac) o non ( $<^{*}$ ne oinom; formas antiguas son noenum, noenu $\left.{ }^{1}\right)$, que se usa para negar el modo de la realidad -indicativo- y la proposición prificipal (aunque también se encuentra en ocasiones delante del subjuntivo con valor condicional). En época imperial non tiende a sustituir a ne en formas como dummodo non, dum non, colocándose asimismo ante algunas palabras negativas (non-nihil, non-numquam, nonnemo, non-nullus), bien para reforzar la negación en casos en los que se había debilitado, bien -otras veces - para producir una lítote (nonnulli 'algunos') ${ }^{2}$. Necnon es una partícula compuesta de dos negaciones, que se emplea para dar mayor fuerza a una negación: En época de Cicerón todavía se encuentran separados los dos elementos; posteriormente tienden a soldarse, y el sentido de la negación así soldada va debilitándose hasta el punto de volverse sinónimo de quoque, etiam

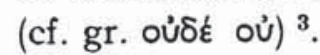

Nonne es una partícula interrogativa que supone una respuesta afirmativa. $\mathrm{Y}$ necne una partícula interrogativo-dubitativa utilizada generalmente en el segundo miembro de una interrogación doble, sobre todo en el estilo indirecto.

$N i$ (antiguo nei), formado sobre ne con el deíctico $-i$ (presente también en haec < *ha-i-ce), tenía en principio el valor de 'no', sin función subordinante; pero se polarizó pronto para acompañar a las condicionales en oposición a si, resultando de este modo sinónimo de nisi (ne $+s i)$

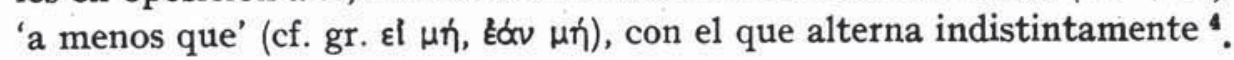

1 La formación de non es exactamente comparable a la de nullum (ant. *ne oinolom) o de nihil (*ne hilom). La caida de -um es la misma que en este último y se explica por la misma razón (vid. DELL, s. u. non).

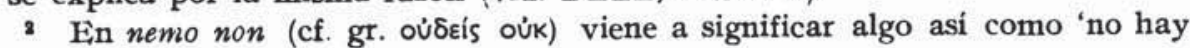
nadie que no'.

3 Vid., entre otros, DELL, s. u. necnon.

- Posteriormente, ni fue eliminado en favor de nisi, forma más plena y que en hiato no se prestaba a equívocos. Nisi en lenguaje familiar habia visto debilitarse su valor condicional en sentido restrictivo: 'solamente, excepto, si no'; por ello se ha reforzado en ocasiones en non nisi (escrito junto o separados) 'no... si no', compañando a ablativos absolutos. 


\subsection{2.}

$\mathrm{Ne}$ se antepone a partículas diversas, especialmente a aquellas que indican tiempo y lugar, para darles valor negativo.

Nedum se utiliza como refuerzo (duim indica simultaneidad) sobre una negación expresada precedentemente: 'con más razón no, todavía menos'. No es subordinante, pero, como el vocablo implica una imposibilidad, a menudo acompaña al subjuntivo. Se empleaba a continuación de negaciones atenuadas o de frases de sentido negativo, pero luego, cuando sus elementos se hicieron inseparables, perdió ese carácter negativo y pasó a ser un mero refuerzo afirmativo. Nequedum y necdum tienen el valor de 'todavía no'.

Nequam, formada de ne y la partícula indefinida quam (cf. perquam, quisquam) se utilizó primero como adverbio, acompañando a esse en el mismo sentido que nihil esse 'no valer nada', y luego como epíteto opuesto a frugi bonae ${ }^{1}$, aunque el empleo adverbial subsiste en locuciones como nequam facere, nequam habere (expresiones en las que Cicerón sustituye a nequam por su derivado nequiter ${ }^{2}$ ).

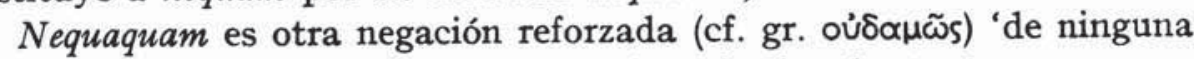
manera, en modo alguno', con un valor afectivo bastante raro, pero atestiguado en todas las épocas ${ }^{3}$.

Nequiquam es un adverbio que tiene el mismo sentido que frustra 'en vano'; procede de $n e+$ un antiguo ablativo en $-i$ del neutro de quisquam, y su valor no es propiamente negativo, aunque se encuentra un recuerdo de ese origen en el hecho de no ser empleado nunca con una negación. Es raro entre los juristas y, al igual que el anterior, ha dejado de usarse bastante tempranamente ${ }^{4}$.

Aparte de estas partículas, podemos mencionar también aquí a numquam $<$ ne + umquam 'nunca' y nequando 'en ningún tiempo', ambas con valor temporal; neutiquam 'de ningún modo' (arcaica y no atestiguada ya después de Tito Livio ${ }^{5}$ ); y las locativas necubi (cf. alicubi,

1 Cif. DELL, s. u. frux.

2 Sobre nequam se ha creado un comparativo nequior y un superlativo nequissimus, a partir de los cuales la lengua popular ha rehecho nequuus (atestiguado en las glosas y confirmado por sus representantes románicos: vid. W. MeyerLübke, Romanisches etymologisches Wörterbuch, Heidelberg, 1935, 5895). Y de esta partícula derivan también el adverbio nequiter y el sustantivo nequitia (cf. $D E L L$, s. u. nequam).

Sid., por ejemplo, Forcellini III, pp. 360-36r.

- Ibidem III, p. $36 \mathrm{I}$.

- Cf. Forcellini III, p. 365. 
sicubi) 'para que en ninguna parte' y necunde (cf. alicunde, undecunde) 'para que de ninguna parte'.

Nimirum es una antigua frase nominal ('no es asombroso') que se ha mantenido como adverbio, y en la que $n i$ conserva su valor de negación sin más (vid. supra) ${ }^{1}$.

\subsection{Ne + pronombres}

$\mathrm{Ne}$ - se antepone también a algunos pronombres indefinidos para negarlos.

Así, ne(c)uter (cf. gr. oúठÉtepos) 'ninguno de los dos, ni el uno ni el otro' (/v/ uter 'cuál de los dos, el de los dos que'), que sirve para designar, en gramática, a los nomina neutra ('que no son ninguno de los dos [géneros]') y que ha sido sustituido por nullus en los autores vulgares de época tardía ${ }^{2}$. Una prueba de que era primitivamente yuxtapuesto (es decir, dos palabras independientes) es que todavía era trisilábico en Plauto ${ }^{3}$. Un derivado de neuter es neutrubi (raro) 'ni en un sitio ni en otro' (cf. utrubi $<$ uter).

Nullus ( $<n e+$ ullus) tiende a sustituir a nemo desde los tiempos más antiguos en algunos casos y a eliminarlo de la lengua hablada. Puede unirse como una especie de aposición a un sujeto expresado o no y al verbo de la frase. En la forma neutra se emplea, a veces, a modo de negación reforzada ${ }^{4}$. Derivados de nullus son: el verbo nullificare, utilizado en la lengua de la Iglesia, nullatenus 'de ninguna manera'

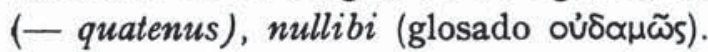

Ningulus parece ser una formación analógica sobre singulus, atestiguada solamente en Ennio y Marcio ${ }^{5}$.

\subsection{Ne + sustantivos}

Nemo procede de ne + homo, pero la relación con éste ha llegado a borrarse hasta el extremo de que nemo es reforzado a menudo por homo en el lenguaje familiar (cf. Plaut., Pers. 2II, Nemo homo usquam

1 Cf. $L E W$ II, s. u. nimirum; DELL, s. u. ni y mirus. Para nimis parece un poco aventurada la hipótesis ni + minus (vid. $L E W$ II, s. u. nimis; $D E L L$, s. u. nimis),

- Tomamos el dato de $D E L L$, s. u. neuter.

Vid. Forcellini III, pp. 364-365.

- Vid. DELL, s. u. nullus.

s Cf. Forcellini III, p. 373; DELL, s. u. ningulus. 
arbitratust) $^{1}$. Puede ir también acompañado de un indefinido (nemo quisquam, nemo unus). Nemo es antiguo y usual, pero, debido a no ser ya analizable en latín, tiende a ser reemplazado por nullus (vid. supra).

$\mathrm{Ne}$ fas $^{2}$ era una frase de tipo nominal, cuyos elementos se han soldado en nefas, como en necessis, necesse. Derivados suyos son: nefastus 'no autorizado por la ley divina o el derecho religioso' (fastus 'autorizado'), aplicado a los días; nefarius, influenciado sin duda por fari y por formas como iniurius $<$ ius.

Negotium es un sustantivo que procede probablemente de frases del tipo mihi neg otium est ${ }^{3}$; es, por lo tanto, el 'no ocio' > 'ocupación, asunto', y también 'dificultad, obstáculo' 4.

En Ennio y Lucrecio aparece neque hilum ( $>$ nihilum $>$ nilum), forma reforzada de la negación (*ne hilom). La elisión de la final en nihil (grafía etimológica) se ha generalizado en virtud de la tendencia a abreviar las palabras accesorias. Pero nihilum, nilum, significando 'la nada', o empleadas con sentido fuerte, conservan la forma plena.

3.

De este análisis somero de las formaciones en las que interviene necomo prefijo, se puede llegar a las siguientes deducciones, interesantes para su comprensión y, por extensión, para el estudio de los prefijos negativos en general:

$\left.{ }^{\text {I. }}{ }^{\mathbf{a}}\right) \mathrm{Ne}$ - (y, en su caso, sus derivados $n i$, non $\left.^{6}\right)$ no es propiamente un prefijo, sino la forma plena de la negación (generalmente con vocalismo breve) que, debido a la frecuencia con que aparece antepuesto

1 Al respecto, cf., por ejemplo, la expresión francesa au jour d'aujourd'hui. Véase J. B. Hofmann, El latín familiar, Madrid, 1958, p. 138.

2 Fas es una palabra del tipo de ius, mos, empleadas en locuciones impersonales.

3 Cf. Paul. Fest. 185,5 negotium quod non sit otium.

- En la lengua hablada se emplea generalmente como 'cosa, asunto' (gr. $\pi \rho \tilde{\gamma} \gamma \mu \alpha)$ y, por eufemismo, se usa para designar cosas o actos que no se quieren nombrar.

- El sentido preciso de hilum, hilo es poco conocido. Se emplea como partícula con el sentido de 'aunque sea poco'. Lo más frecuentemente van acompañados de negación. Nihil es una palabra expresiva en época antigua, pero posteriormente ha perdido su valor y está mal atestiguada en romance (vid. DELL, s. u. hilum y nihil).

- Non es quizás, de todas estas formas, la que menos se ha soldado a la palabra que sigue, tal vez porque ha comenzado a usarse con el valor de ne en un momento más tardío, cuando ya in-se mostraba suficiente para el uso prefijal. 
a determinadas palabras (cf., por ejemplo, nequeo o neuter), ha terminado por fundirse con ellas hasta llegar a ser en algunos casos casi inanalizable (necesse, nolo, etc.) ${ }^{1}$.

2.8) Todos los casos analizados son formaciones antiguas: en época clásica ne estaba ya soldado a todos los términos con que aparece, y desde entonces no ha vuelto a funcionar como prefijo dentro del sistema lingüístico latino, debido, tal vez, y sobre todo, a la competencia de in-, forma reducida de la misma negación y, por lo tanto, propia para formar vocablos negativos.

$3 .{ }^{\text {a) }}$ Por otra parte, - funciona especialmente antepuesto a verbos, sustantivos, indefinidos y partículas (es decir, con aquellos tipos de palabras a los que, en principio, no puede anteponerse $i n-)^{2}$. Y, en cambio, no se encuentra con adjetivos, excepto cuando éstos derivan de un verbo con ne- (nescius, neglectus); el que en algunos casos no esté registrada esta base verbal con ne- da lugar a dos posibilidades de explicación: a) o bien las formas verbales de las que derivan los adjetivos eran usadas a menudo con negación, de tal modo que han podido llegar a sentirse como una sola palabra; b) o bien habría que admitir la posibilidad de que ne- funcionara también con adjetivos.

Pero, en contra de esta segunda hipótesis, está el hecho de que son muy pocos los casos discutibles, y de ellos necopinatus y necopinans son formas verbales, aunque no exista *necopinor; necopinus procede de ellas por retrogradación. En cuanto a nesapius, podría aceptarse plenamente la primera posibilidad, atendiendo además a la sinonimia con nescio, nescius. Para nefandus y nefans hay que tener en cuenta, no sólo a fari, sino también a nefas.

Todo lo anterior puede resaltarse un tanto si establecemos una rápida comparación con in-, puesto que $n e$ - e $i n$ - no son en realidad más que dos variantes de una misma cosa: la partícula negativa, en este caso aglutinada con determinados vocablos. Recordemos al respecto que, si bien el valor de ne- como prefijo y el de in- es el mismo (negar el contenido de la base a la que se anteponen), in- es única y exclusivamente prefijo (no aparece como forma autónoma, como no podía menos de ser dado su origen) ${ }^{8}$, y funciona como tal en época antigua (igual que ne-), pero, al contrario que éste, no agota ahí sus posibilidades, sino

1. Cf. supra nefas, negotium: frases nominales $>$ sustantivos negativos.

- Por regla general, los sustantivos y verbos que llevan in- derivan de adjetivos que ya presentaban este prefijo (incapacitas < incapax; indeceo < indecens).

- Conviene no olvidar que existen dos formas homófonas: in (preposición y prefijo locativo-direccional) e in- (prefijo negativo). 
EN TORNO A ne- COMO PRIMER ELEMENTO DE UNA SERIE DE VOCABLOS 159

que permanece vigente durante toda la latinidad, dando lugar a una gran cantidad de vocablos en todas las épocas, y de un modo especial en latín tardío, sobre todo en la lengua de la Iglesia y en lenguajes técnicos en general (ineffabilis, inabstractus, intransitivus, etc.) ${ }^{1}$.

$\mathrm{Ne}$-, por el contrario, es primitivamente una aglutinación que tiende a lexicalizarse en una serie de casos, como lo prueba el hecho de que, cuando se desea recalcar la negación, se deba recurrir a non (cf. neuis > non uis).

\section{MERCEDES BREA}

Santiago de Compostela

1 Por otra parte, in- se utiliza para negar adjetivos, y ne- no (vid. supra). 